\title{
Role of Calcium in Hypertensive Disorders of Pregnancy Current Status of Research a Mini Review
}

\section{Shakuntala Chhabra* and Anupria Singh}

Department of Obstetrics Gynaecology, Mahatma Gandhi Institute of Medical Sciences, Maharashtra, India

*Corresponding author: Shakuntala Chhabra, Director Professor, Department of Obstetrics Gynecology, Mahatma Gandhi Institute of Medical Sciences, Maharashtra, India, Tel: 91-7152-284342; E-mail: schhabra@mgims.ac.in

Received date: April 19, 2017; Accepted date: May 12, 2017; Published date: May 19, 2017

Copyright: ( 2017 Chhabra S, et al. This is an open-access article distributed under the terms of the Creative Commons Attribution License, which permits unrestricted use, distribution, and reproduction in any medium, provided the original author and source are credited.

\begin{abstract}
Introduction: Some studies have revealed changes in blood/urinary calcium with inverse relationship between calcium intakes during pregnancy, incidence of preeclampsia. So researchers believe that calcium has a role in etiology of hypertensive disorders during pregnancy. With reduced incidence of preeclampsia after calcium supplementation. So prevention of HDsP and/or their severity are being attempted through oral calcium.
\end{abstract}

Objective: To collect existing information about role of calcium in HDsP for translational program or if needed, research.

Material Methods: Present article is based on literature search by available search engines like, Pubmed, Google, Update and others. Studies, reviews and short commentaries were looked into to get desired information.

Serum urinary cellular calcium: Normally there is increase in intracellular calcium in pregnancy. It has been reported that this effect is exaggerated in preeclampsia due to significant increase in the membranous calcium content. Researchers also reported that preeclampsia in third trimester was associated with hypocalciuria due to increased tubular reabsorption of calcium. Urinary calcium excretion has been found to be reduced in severe preeclampsia, eclampsia, but decrease could not be used to identify severity of preeclampsia, or predict impending eclampsia. Problem seems to be at the cellular level, needs to be investigated further.

Calcium supplementation: Some have reported incidence of preeclampsia greater in women given placebo, compared to calcium-treated, although difference was not significant. Also supplementing calcium in women at high risk of pre-eclampsia was likely to reduce incidence of disease. But others reported that extra calcium did not reduce number of women who suffered, however reduced severity of HDsP. Also smooth-muscle relaxation detected in calcium supplemented women might affect labour onset.

Conclusion: There are reports of positive relationship and no effect on reduction in HDsP or their severity. In view of contradictory findings, in depth studies are required to investigate altered serum, urine and cellular calcium, in women with HDsP.

Keywords: Calcium; Supplementation; Hypertensive disorders during pregnancy; Severity

\section{Introduction}

Over the years researchers have suggested that calcium may be playing a role in the etiology, of hypertensive disorders during pregnancy. Some studies have revealed changes in blood/urinary calcium in women with HDsP [1-3]. So prevention of HDsP and their severity are being attempted through calcium supplementation [4-11]. Hofmeyr et al. [12] have reported the impairment of calcium metabolism with low circulating vitamin $\mathrm{D}$ concentration and/or inadequate dietary calcium which may be contributing to the risk of HDsP. After various clinical trials, some researchers [4,5,7,13-16] reported reduced incidence of preeclampsia with calcium supplementation, others reported association of lower blood pressure, with higher dietary calcium [6]. Epidemiologic studies have revealed an inverse relationship between calcium intake during pregnancy and the incidence of preeclampsia [12]. However there are contradictory reports also [17]. So research continues.

\section{Objective}

To collect information about the role of calcium in HDsP for planning its use as translational program or for more research.

\section{Material Methods}

Present article is based on literature search by available search engines like, Pubmed, Google, Update and others for looking into studies, reviews and short commentaries to get desired information as per the objectives. 


\section{Status}

\section{Calcium in women with normal pregnancy and pregnancy with hypertensive disorders:}

Normally there is an increase in intracellular calcium in pregnancy. The alterations in calcium metabolism responsible for the pathogenesis of HDsP, were earlier studied by Frolich et al. [18]. Normotensive pregnant women and pre-eclamptic women exerted distinct changes on cellular $\mathrm{Ca} 2+$ metabolism in normal vascular smooth muscle cells (VSMC) [19]. Normal pregnant sera amplified, whereas preeclamtic sera blunted the volgate dependent calcium channels (VDCC). LopezJamiloo [15] reported that the calcium intake in women with preeclampsia was significantly lower with low levels of the serum ionized calcium. Kisters et al. [20] have reported that changes in intracellular calcium and magnesium concentration were involved in the pathogenesis of preeclampsia and the role of cell membranes has not been studied well. Researchers did a study to investigate the changes in plasma calcium and magnesium metabolism, intracellular and membrane calcium and magnesium concentrations in women with normal pregnancy and those with preeclampsia and found that membranous calcium content was significantly increased in the preeclamptic cases compared to controls or healthy pregnant women and an inverse correlation with membranous magnesium content was found. Hovdenak [21] has reported calcium deficiency associated with preeclampsia and intrauterine growth restriction. It has been reported that this effect is exaggerated in preeclampsia due to a significant increase in the membranous calcium content $[7,22,23]$. Conversely Kisters et al. [20] reported low membranous and intracellular calcium concentration in women with HDsP compared to healthy pregnant women. Membranous calcium content was significantly increased in the women with preeclampsia compared to nonpregnant or healthy pregnant controls. Green et al. [24] suggested the presence of circulatory serum factor with $\mathrm{Ca}(2+)$ in $\mathrm{HDsP}$, major determinant of VSMC tone. Researchers reported that it was possible that consequent to the attenuation of $\mathrm{Ca}(2+)$ responses. This factor counter balanced the intense vasoconstriction seen in HDsP. Decades back, Pitkin et al. [25] reported increased calcium absorption in normal pregnancy, but serum calcium did not change significantly, because urinary calcium excretion was around 350-620 mg/day during pregnancy compared to $100-250 \mathrm{mg} /$ day in normal women. But urinary calcium was considerably reduced in women with preeclampsia and hence hypocalciuria could serve as a predictive and prognostic marker for HDsP. Seely et al. [26] reported relative hypocalciuria in women with preeclampsia in third trimester. Researchers also reported that women with HDsP had lower serum ionized calcium than in normotensive pregnant women in third trimester. The findings confirmed that the women with preeclampsia had relative hypocalciuria. Anai et al. [27] also reported that calcium excretion was significantly lower in severe as well as mild preeclampsia cases, than normal pregnant women with a significant difference between the values in the mild and severe cases also. Pederson et al. [28] also reported fractional excretion of calcium in HDsP lower than normotensive pregnant women in the third trimester. Later Taufield et al. [29] reported that the serum ionized calcium was not different in third trimester amongst normal pregnancy, chronic hypertension, chronic hypertension with superimposed pre-eclampsia, transient hypertension and preeclampsia, but the mean 24-hour urinary calcium excretion in the women with preeclampsia or hypertension with superimposed preeclampsia were significantly lower than normal pregnant women, women with transient hypertension, or women with chronic hypertension. The women with preeclampsia had lower fractional excretion of calcium. The researchers concluded that preeclampsia in third trimester was associated with hypocalciuria due to increased tubular reabsorption of calcium. Hence measurement of calcium excretion could be useful for distinguishing preeclampsia from other forms of hypertension. Varner et al. [30] were the first to report that, calcium metabolism in term pregnancy associated with essential hypertension without other complications, was characterized by significantly reduced levels of ionized calcium. Sanchez-Ramos et al. [31] also reported that the women with preeclampsia had significantly less excretion of total calcium than normotensive women or those with gestational hypertension. The study revealed that urinary calcium below $12 \mathrm{mg} / \mathrm{dL}$ might help in distinguishing preeclampsia from other forms of hypertension with positive and negative predictive values, $85 \%$ sensitivity and $91 \%$, specificity respectively. Myatt et al. [32] reported that an increase in intracellular calcium in VSMC during pregnancy was consistent with development of vasoconstriction and resultant hypertension. Earlier Ray et al. [2] had reported that normotensive women during the third trimester and women with chronic hypertension had significantly higher basal intracellular, free calcium concentration than normal pregnant women during the first trimester and preeclampsia was associated with increased levels of intracellular calcium, decreased calcium-dependent ATPase activity of erythrocytes and hypocalciuria. VanWijk [33] reported increased vascular tone of subcutaneous arteries in preeclampsia associated with an increased sensitivity to intracellular calcium. Vural et al. [34] reported that in preeclampsia, alterations in renal function, electrolytes and water metabolism were common findings and that preeclampsia was associated with hypocalciuria, but there was no correlation between parameters of renal function and calcium or phosphate excretion. Ingec et al. [35] reported that low levels of urinary calcium excretion were due to increased tubular reabsorption of calcium. Golmohammadlou et al. [36] have reported no significant difference in serum calcium and the researchers reported that the trace element of calcium was not involved in the pathogenesis of preeclampsia. However Jafrin et al. [37], reported serum calcium, significantly less in HDsP, compared to normal pregnant women.

\section{Urinary/Serum calcium as diagnostic/predictor of HDsP:}

Xie et al. [38] reported that the urinary calcium excretion could be used as an indicator for HDsP from a single void urine sample or 24 hour urinary calcium excretion. Segovia et al. [39] reported hypocalciuria, a significant factor in preeclampsia or transitory hypertension in third trimester of pregnancy. Ingec et al. [35] also reported significantly lower 24 hour urinary calcium levels in women with mild, severe preeclampsia and eclampsia than controls. Urinary calcium levels between mild and severe pre-eclampsia and eclampsia were similar, but were lower in eclampsia than in mild preeclampsia. The study revealed that, urinary calcium excretion was reduced in patients with severe preeclampsia and eclampsia, but researchers reported that the decrease could not be used to identify the severity of preeclampsia, or to predict impending eclampsia. Dasgupta et al. [40] have reported the changes in calcium excretion in pre-eclampsia, eclampsia and their role as predictor and concluded that hypocalciuria was a good tool for prediction of HDsP and was independent of renal function. Consistent with this study, another study done by Jafrin et al. [37] revealed significant decrease in serum calcium in subjects with pre-eclampsia compared to normal pregnant women. Sirohiwal et al. [41] reported that compared to normotensive women, the hypertensive 
women had significantly lower urinary calcium excretion in the study which was done to know the efficacy of measurement of 24 hour urinary protein and calcium for the prediction of preeclampsia in second trimester. It was found that a decrease in 24 hour urinary calcium between 20-28 weeks gestation was predictive of preeclampsia later. Urinary calcium and calcium creatinine levels in women destined to develop PE progressively diminished till term, similar to findings of several researchers [31,36,42-43]. Dasgupta [44] reported that although the physiological calciuria of the controls could be explained by an increased Glomerular filtration rate (GFR), the fall in calcium excretion in preeclampsia subjects was independent of the glomerulopathy and altered filtration rate. The problem seems to be at the cellular level and needs to be investigated further.

\section{Calcium supplementation:}

The idea, whether calcium supplement would help reduce the incidence of HDsP, was first thought of because of the findings of an epidemiological study which revealed that Mayan Indians exhibited high calcium intake and low incidence of preeclampsia and eclampsia [45]. Marcoux et al. [46] reported that calcium intake during pregnancy was inversely related to the risk of gestational hypertension but not preeclampsia. Villar et al. [47] in their study provided women with 2.8 gms of oral calcium per day from the twenty third week of pregnancy and reported a lower incidence of preterm pains (less than 37 weeks; $7.4 \%$ vs. $21.1 \%$ spontaneous preterm delivery, $6.4 \%$ vs. $17.9 \%$ and low birth weight $(9.6 \%$ vs. $21.1 \%)$. The observed effect could be mediated by a reduction in uterine smooth muscle contractibility. Repke et al. [48] also reported that the incidence of preeclampsia was greater in women given placebo than those treated with calcium, although this difference was not significant. The study not only revealed the calcium influence on the incidence and/or gestational age at development of HDsP, but also the smooth-muscle relaxation detected in calcium-supplemented patients. Marcoux et al. [47] in their case-control study assessed the relation of calcium intake in the first 20 weeks of pregnancy to the risk of preeclampsia, gestational hypertension and reported that calcium intake during pregnancy may be inversely related to the risk of gestational hypertension. In rural Guatemala, despite the low socioeconomic status and low intake of protein and energy of women, the incidence of eclampsia was low and the researcher [48] reported that dietary calcium in this population was relatively high, in large part because of the incorporation of limeprocessed tortillas as a staple component of the diet. Cong et al. [49] reported that when calcium was given daily from 20 to 28 weeks of gestation up to delivery, the incidence of $\mathrm{HDsP}$ was $4 \%$, while in controls it was $18 \%$. Supplementation of $2 \mathrm{~g}$ of calcium daily resulted in lowering the incidence of HDsP significantly without any adverse effects. Similarly Zemel et al. [50] reported that sub-optimal calcium intake contributed to the change in salt-sensitivity and hypertension and high salt diets exerted a calciuretic effect. Herrera et al. [8] reported significantly reduced incidence of HDsP in women who received the supplement with decreased intracellular concentration of ionized free calcium in peripheral blood lymphocytes.

Imdad et al. [11] reported an extra gain in fetal weight by $85 \mathrm{gms}$ in cases of HDsP, in women who were supplemented with calcium compared with controls though there was no difference in perinatal mortality. These data suggested the possible altered vascular and cellular sensitivity of endothelium to calcium which lead to the cascade of placental vascular insufficiency and fetal growth restriction. Association between lower blood pressure with higher levels of dietary calcium was reported earlier too [6]. Epidemiological studies, laboratory evaluations, and clinical trials also indicated that the incidence of HDsP was affected by calcium intake [9]. But most trials have not assessed the possibility that a subgroup with low baseline urinary calcium may benefit most from calcium supplementation https://clinicaltrials.gov/ct2/show/NCT00000534.

Chen et al. [51] reported that high levels of calcium available to endothelial cells prior to their exposure to the preeclampsia-associated activators could reduce the endothelial cell activation. The studies revealed that additional calcium treatment did prevent endothelial cell activation, in response to activators induced by low calcium. World Health Organization [52] also reported that calcium could reduce severity of preeclampsia, though did not seem to reduce the number of women who suffered from this condition. In populations with low dietary calcium intake, daily $1.5 \mathrm{~g}-2.0 \mathrm{~g}$ oral elemental calcium has been recommended to reduce the risk of pre-eclampsia. This recommendation was consistent with the 'WHO recommendations for prevention and treatment of pre-eclampsia and eclampsia' [53] and superseded the other recommendation found in the WHO guideline 'Calcium supplementation in pregnant women' [54].

Earlier Villar [55] reported that $1.5 \mathrm{~g}$ calcium/day supplement did not prevent preeclampsia but did reduce its severity, maternal morbidity, and neonatal mortality. Hovdenak [21] reported that supplementation reduced both the risk of LBW and the severity of preeclampsia. Review by Buppasiri et al. [56] revealed that calcium supplementation was associated with a significant benefit in the prevention of pre-eclampsia. Review of data from 10 randomized controlled trials reviewed by Imdad et al. [57] revealed that calcium supplementation during pregnancy was associated with a reduction in risk of gestational hypertension, pre-eclampsia, neonatal mortality in developing countries. Results of meta-analysis by Patrelli et al. [10] revealed that the additional intake of calcium during pregnancy was an effective measure to reduce the incidence of preeclampsia, especially in populations at high risk of preeclampsia. Carroli [58] reported that calcium supplementation of pregnant women with deficient calcium intake may affect uteroplacental and fetoplacental blood flow, by preserving the vasodilation of normal gestation. Imdad [11] also reported that calcium supplementation during pregnancy was associated with a reduction in risk of gestational hypertensive disorders and pre-term birth and an increase in birth weight. The review of 24 trials found good quality evidence that calcium supplementation with high doses (at least $1 \mathrm{~g}$ daily) during pregnancy (13 studies involving 15,730 women) was a safe and relatively cheap way of reducing the risk of pre-eclampsia, especially in women from communities with low dietary calcium and those at increased risk of pre-eclampsia. Others also reported Calcium supplementation improved calcium intake and consequently reduced the risk of hypertensive disorders during pregnancy [59]. Women receiving calcium supplements were also less likely to die or have serious problems related to pre-eclampsia.

Recent systematic reviews and meta-analyses have reported that pregnant women taking at least 1 gram of supplemental calcium daily reduced their risk of developing HDsP disorders by approximately half, with the effect varying largely by habitual calcium consumption and underlying risk of preeclampsia. The largest protective effects were found in populations with habitual calcium consumption less than 900 mg. Low habitual calcium intake was defined as, 'average habitual calcium intake less than $900 \mathrm{mg}$ of calcium per day'. To illustrate this amount in terms of foods, $900 \mathrm{mg}$ calcium could be provided by 3 cups 
$(750 \mathrm{~mL})$ of milk, or three servings of hard cheese such as cheddar, or 9 cups (more than $2 \mathrm{~L}$ ) of cooked greens such as kale [60].

Limited evidence from 10 trials (2234 women) suggested that a relatively low dose may be effective. In settings of low dietary calcium where high-dose supplementation were not feasible, the option of lower dose supplements ( 500 to $600 \mathrm{mg} /$ day) might be considered in preference to no supplementation [61]. Richards et al. [62] in an observational case control study reported that supplementing calcium in pregnant women at high risk of developing pre-eclampsia was likely to reduce the incidence of the disease. So they measured differences in serum and hair concentrations of calcium and magnesium of women with pre-eclamptic and normotensive pregnancies but there was no significant difference in the hair calcium between pre-eclamptic and normotensive pregnancies and the study revealed that calcium supplementation did not reduce the risk of developing pre-eclampsia. Though no adverse effects have been found but further research was needed into role of calcium and dose.

\section{Conclusion}

Over all studies have supported as well as negated the hypothesis of hypocalcemia/ hypocalciuria with HDsP. The findings of various studies may also be different, because of timing of sample collection, number of samples, storage and processing, prior history of calcium intake and level of dietary calcium. In view of the contradictory findings more indepth studies are required to investigate about the altered calcium mechanisms, calcium excretion in women with HDsP. More studies are needed about calcium homeostatis in women with HDsP, studies to know the role of calcium in the occurrence and in the severity HDsP, specially studies about cellular level calcium.

\section{References}

1. Zemel MB, Zemel PC, Berry S, Norman G, Kowalczyk C, et al. (1990) Altered platelet calcium metabolism as an early predictor of increased peripheral vascular resistance and preeclampsia in urban black women. $\mathrm{N}$ Engl J Med 323: 434-438.

2. Ray J, Vasishta K, Kaur S, Majumdar S, Sawhney H (1999) Calcium metabolism in pre-eclampsia. Int J Gynaecol Obstet 66: 245-250.

3. Farzin L, Sajadi F (2012) Comparison of serum trace element levels in patients with or without pre-eclampsia. J Res Med Sci 17: 938-941.

4. Carroli G, Duley L, Belizan J M, Villar J (1994) Calcium Supplementation during Pregnancy: A Systematic Review of Randomised Controlled Trials. Br J Obstet Gynaecol 101: 753-758.

5. Bucher HC, Guyatt GH, Cook RJ, Hatala R, Cook Dj, et al. (1996) Effect of calcium supplementation on pregnancy-induced hypertension and preeclampsia: a meta-analysis of randomized controlled trials. JAMA 275: 1113-1117.

6. Food and Nutrition Board, I. O. M, National Academy Of Sciences (1997) Dietary reference intakes (dris) for calcium, phosphorus, magnesium, vitamin d, and fluoride. Washington, Dc: National Academy Press.

7. Ritchie LD, King JC (2000) Dietary calcium and pregnancy-induced hypertension: Is there a relation? Am J Clin Nutr 71: 1371s-1374s.

8. Herrera JA, Arevalo-Herrera M, Shahabuddin A K, Ersheng G, Herrera S, et al (2006) Calcium and conjugated linoleic acid reduces pregnancyinduced hypertension and decreases intracellular calcium in lymphocytes. Am J Hypertens 19: 381-387.

9. Hofmey GJ, Lawrie TA, Atallah AN, Duley L (2010) Calcium supplementation during pregnancy for preventing hypertensive disorders and related problems. Cochrane database Syst Rev Cd001059.

10. Patrelli TS, Dall'asta A, Gizzo S, Pedrazzi G, Piantelli G, et al. (2012) Calcium supplementation and prevention of preeclampsia: a metaanalysis. J Matern Fetal Neonatal Med 25: 2570-2574.
11. Imdad A, Bhutta ZA (2012). Effects of calcium supplementation during pregnancy on maternal, fetal and birth outcomes. Paediatr Perinat Epidemiol 26: 138-152.

12. Hofmeyr GJ, Lawrie TA, Atallah AN, Duley L, Torloni MR (2014) Calcium supplementation during pregnancy for preventing hypertensive disorders and related problems. Cochrane Database Syst Rev 6: CD001059

13. Ito M, Koyama H, Ohshige A, Maeda T, Yoshimura T, et al. (1994) Prevention of preeclampsia with calcium supplementation and vitamin d3 in an antenatal protocol. Int J Gynaecol Obstet 47: 115-120.

14. Lopez-Jaramillo P (1996) Prevention of preeclampsia with calcium supplementation and its relation with the l-arginine: nitric oxide pathway. Braz J Med Biol Res 29: 731-741.

15. Lopez-Jaramillo P (2000) Calcium, nitric oxide, and preeclampsia. semin perinatol 24: 33-36.

16. Trumbo PR, Ellwood KC (2007) Supplemental calcium and risk reduction of hypertension, pregnancy-induced hypertension, and preeclampsia: an evidence-based review by the us food and drug administration. Nutr Rev 65: 78-87.

17. Burnell AM, Tunnacliffe A, Perry RN, Wharton DA (2011) Molecular and physiological basis of nematode survival. wallington: cabi. gene induction and desiccation stress, UK

18. Frolich A, Rudnicki M, Storm T, Rasmussen N, Hegedus L (1992) Impaired 1,25 dihydroxyvitamin $\mathrm{d}$ production in pregnancy-induced hypertension. Eur J Obstet Gynecol Reprod Biol 47: 25-29.

19. Swapna V, Jambale T, Murthy J (2015) Study of urinary calcium and urinary creatinine levels and urinary calcium/creatinin ratio in gestational hypertensive patients. J of Evolution of Medical and Dental Sciences 4: 9145-9150.

20. Kisters K, Barenbrock M, Louwen F, Hausberg M, Rahn KH, et al. (2000) Intracellular, and plasma magnesium and calcium concentrations in preeclampsia. Am J Hypertens 13: 765-769.

21. Hovdenak N, Haram K (2012) Influence of mineral and vitamin supplements on pregnancy outcome. Eur J Obstet Gynecol Reprod Biol. 164: 20127-20132.

22. Kosch M, Hausberg M, Louwen F, Barenbrock M, Rahn KH, et al. (2000) Alterations of plasma calcium and intracellular and membrane calcium in erythrocytes of patients with pre-eclampsia. J Hum Hypertens 14: 333-336.

23. Heringhausen J, Montgomery KS (2005) Continuing education modulematernal calcium intake and metabolism during pregnancy and lactation. J Perinat Educ 14: 52-57.

24. Green J, Assady S, Nakhoul F, Bick T, Jakobi P, et al. (2000) Differential effects of sera from normotensive and hypertensive pregnant women on $\mathrm{ca}(2+)$ metabolism in normal vasular smooth muscle cells. J Am Soc Nephrol 11: 1188-1198.

25. Pitkin RM (1975) Calcium metabolism in pregnancy: a review. Am J Obstet Gynecol 121: 724-737.

26. Seely EW, Wood RJ, Brown EM, Graves SW (1992) Lower serum ionized calcium and abnormal calciotropic hormone levels in preeclampsia. J Clin Endocrinol Metab 74: 1436-1440.

27. Anai T, Hirota Y, Yoshimatsu J, Oga M, Miyakawa I (1992) Hypocalciuria in women with preeclampsia. nihon sanka fujinka gakkai zasshi 44: 28-32.

28. Pedersen EB, Johannesen P, Kristensen S, Rasmussen AB, Emmertsen K, et al. (1984) Calcium, parathyroid hormone and calcitonin in normal pregnancy and preeclampsia. Gynecol Obstet Invest 18: 156-164.

29. Taufield PA, Ales KL, Resnick LM, Druzin ML, GertnerJM, et al. (1987) Hypocalciuria in preeclampsia. N Engl J Med 316: 715-718.

30. Varner MW, Cruikshank DP, Pitkin RM (1983) Calcium metabolism in the hypertensive mother, fetus, and newborn infant. Am J Obstet Gynecol 147: 762-765.

31. Sanchez-Ramos L, Sandroni S, Andres FJ, Kaunitz AM (1991). Calcium excretion in preeclampsia. Obstet Gyneco 77: 510-513. 
32. Myatt L (1992) The relation of calcium nutrition and metabolism to preeclampsia and premature labor. in: tsang rc, mimouni $\mathrm{f}$, (Eds. Calcium Nutriture For Mothers And Children), Raven Press, New York.

33. Vanwijk M (2000) Vascular function in preeclampsia. Cardiovascular Research 47: 38-48.

34. Vural P, Akgul C, Canbaz M (2000) Calcium and phosphate excretion in preeclampsia. Turk J Med Sci 30: 39-42.

35. Ingec M, Nazik H, Kadanali S (2006) Urinary calcium excretion in severe preeclampsia and eclampsia. Clin Chem Lab Med 44: 51-36.

36. Golmohammad Lou SA, Yazdian A, Pashapour N (2008) Evaluation of serum calcium, magnesium, copper, and zinc levels in women with preeclampsia iran. J Med Sci 33: 231-234.

37. Jafrin W, Paul SK, Sultana S, Rabeya S, Hoque MR, et al. (2013) Serum calcium level among normal pregnant and pre-eclamptic women in a sub urban area of bangladesh. Mymensingh Med J 22: 418-422.

38. Xie Y, Zhang G, Liu X, Qiu W, Li W, et al. (1995) Urinary calcium excretion in patients with pregnant hypertension syndrome. Hua Xi Yi Ke Da Xue Xue Bao 26: 94-97.

39. Segovia BL, Vega IT, Villarreal EC, Licona NA (2004) Hypocalciuria during pregnancy as a risk factor of preeclampsia. Ginecol Obstet Mex 72: 570-574.

40. Dasgupta M, Adhikari S, Sanghamita M (2008) Urinary calcium levels in pre-eclampsia. J Obstet Gynecol India 58.

41. Sirohiwal D, Dahiya K, Khaneja N (2009) Use of 24-hour urinary protein and calcium for prediction of preeclampsia. Taiwan J Obstet Gynecol 48: 113-115.

42. Suarez VR, Trelles JG, Miyahira JM (1996) Urinary calcium in asymptomatic primigravidas who later developed preeclampsia. Obstet Gynecol 87: 79-82.

43. Ramos JG, Martins-Costa SH, Kessler J., Costa CA, Barros E (1998). Calciuria and pre-eclampsia. Braz J Med Biol Res 31: 519- 522.

44. Dasgupta M, Adhikari S, Sanghamita M (2008) Urinary calcium levels in pre-eclampsia. j obstet gynecol india 58: 4.

45. Atallah AN, Hofmeyr GJ, Duley L (2000) Calcium supplementation during pregnancy for preventing hypertensive disorders and related problems. Cochrane Database Syst Rev Cd001059.

46. Marcoux S, Brisson J, Fabia J (1991) Calcium intake from dairy products and supplements and the risks of preeclampsia and gestational hypertension. Am J Epidemiol 133: 1266-1272.

47. Villar J, Repke JT (1990) Calcium supplementation during pregnancy may reduce preterm delivery in high-risk populations. Am J Obstet Gynecol 163: 1124-1131.
48. Repke JT, Villar J (1991) Pregnancy-induced hypertension and low birth weight: the role of calcium. Am J Clin Nutr 54: 237s-241s.

49. Cong K, Chi S, Liu G (1995) Calcium supplementation during pregnancy for reducing pregnancy induced hypertension. Chin Med J (Engl) 108: 57-59.

50. Zemel M (2001) Calcium modulation of hypertension and obesity: mechanisms and implications. J Am Coll Nutr 20: 428s-435s.

51. Chen Q, Tong M, Wu M, Stone PR, Snowise S, et al. (2013) A calcium supplementation prevents endothelial cell activation: possible relevance to preeclampsia. J Hypertens 31: 1828-1836.

52. WHO recommendations on antenatal care for a positive pregnancy experience (2016) Sexual and reproductive health. WHO.

53. WHO recommendations for prevention and treatment of pre-eclampsia and eclampsia (2011) e-Library of Evidence for Nutrition Actions. WHO.

54. Guideline: Calcium supplementation in pregnant women. Geneva (2013) World Health Organization.

55. Villar J, Aleem H, Merialdi M, Mathai M, Ali M, et al. (2006) World health organization randomized trial of calcium supplementation among low calcium intake pregnant women. Am J of Obst\&Gyne 194: 639-649.

56. Buppasiri P, Lumbiganon P, Thinkhamprop J, Ngamjarus C, Laopaiboon M (2011) Calcium supplementation (other than for preventing or treating hypertension) for improving pregnancy and infant outcomes. Cochrane Database Syst Rev 5.

57. Imdad A, Jabeen A, Bhutta ZA (2011) Role of calcium supplementation during pregnancy in reducing risk of developing gestational hypertensive disorders: a meta-analysis of studies from developing countries. BMC Public Health 11: S18.

58. Carroli G, Merialdi M, Wojdyla D, Abalos E, Campodonico L, et al. (2010) Effects of calcium supplementation on uteroplacental and fetoplacental blood flow in low-calcium-intake mothers: a randomized controlled trial. Am J Obstet Gynecol 202: 45e1-9.

59. Calcium supplementation during pregnancy to reduce the risk of preeclampsia (2017) World health Organisation.

60. Calcium supplementation in pregnancy for prevention of pre-eclampsia (2017) Nutritional international.

61. Hofmeyr G, Lawrie TA, Atallah ÁN, Duley L, Torloni MR (2014) Calcium supplementation during pregnancy for preventing hypertensive disorders and related problems. Cochrane.

62. Richards D, Lindow S, Carrara H, Knight R, Haswell S, et al. (2013) A comparison of maternal calcium and magnesium levels in pre-eclamptic and normotensive pregnancies: an observational case-control study. BJOG 121: 327-336. 